\title{
ELECTRON DIFFRACTION
}

\section{P.19.03.1}

Acta Cryst. (2005). A61, C458

Relation of Local and Integral Intensities of Reflections on Oblique Texture Electron Diffraction (OTED) Pattern

Max Nickolsky, Institute of Ore Deposits (IGEM) RAS, Moscow, Russia. E-mail:sir_Maxus@mail.ru

OTED patterns arise from samples with preferred orientation of crystals. Reflections on OTED patterns from plate-like crystals appear as arcs. Structure amplitude could be determined either from integral intensity of reflection (integration of intensity of hole arc) or from local intensity of reflection (integration of intensity along radial profile of arc). Equations of intensity of reflections on OTED patterns were derived by Vainstein [1].

Local intensity is given in [1] as: $I_{h k l}{ }^{\prime}=I_{h k l} \frac{\Delta}{r \alpha}(1)$, where $I_{h k l^{\prime}}$ and $I_{h k l}$ - local and integral intensity of reflection, respectively, r $\alpha-$ azimuthal length of reflection, $\Delta$ - width of profile.

New registration systems (Imaging Plate, CCD), suitable for electron diffraction, allow investigation of shape of reflections on OTED patterns in much more detailed way than ever. As can be seeing directly, distribution of intensity in azimuthal direction of arc is not uniform and thus, equation (1) is not correct.

Precise determination of structure amplitude could be done with the respect to the shape of arc in azimuthal direction. The last depends on deviation of sample from ideal "texture" and could not be predicted in advance. The only way is to describe shape of reflections with analytical functions for each particular sample, assuming that shape of reflection is one and the same for all reflections on OTED pattern.

[1] Vainstein B. K., Kristallografiya, 1957, 2, 340.

Keywords: electron diffraction, imaging plates, texture patterns

\section{P.19.03.2}

Acta Cryst. (2005). A61, C458

Structure Determination of Embedded Precipitates by Precession Electron Diffraction and Synchrotron Radiation: A Comparison Vidar Hansen ${ }^{\mathrm{a}}$, Atle Kverneland ${ }^{\mathrm{a}}$, Helge Bøvik Larsen ${ }^{\mathrm{a}}$, Philip Pattison $^{\mathrm{b}}$, Roger Vincent ${ }^{\mathrm{c}}$, Jon Gjønnes ${ }^{\mathrm{d}}$, ${ }^{\mathrm{a}}$ Department of Mechanical and Structural Engineering and Materials Science, University of Stavanger, N-4036 Stavanger, Norway. ${ }^{\mathrm{b}}$ SNBL-ESRF, 6 Rue Jules Horowitz, BP 220, F-38043 Grenoble Cedex9, France. ${ }^{\mathrm{c}}$ H.H. Wills Laboratory, University of Bristol, Tyndall Ave, Bristol BS8 1TL, United Kingdom. ${ }^{\mathrm{d} C e n t e r ~ f o r ~ M a t e r i a l s ~ S c i e n c e ~ a n d ~ n a n o t e c h n o l o g y, ~}$ University of Oslo, Gaustadalleen 21, N-0349 Oslo, Norway. E-mail: vidar.hansen@uis.no

Phases that appear in industrially important materials are often difficult to prepare in a form suitable for structure determination by standard X-ray methods. The metastable, $\eta^{\prime}$-phase in age-hardened $\mathrm{Al}-\mathrm{Zn}-\mathrm{Mg}$ alloys occurs only as nano-sized semicoherent precipitates embedded in the alloy matrix. Previous X-ray [1], and HRTEM [2] work have not provided a satisfactory structure solution. The $\eta^{\prime}$ structure has therefore been analyzed by us, using three-dimensional electron diffraction data collected from thin-foils prepared from the age-hardened 88.12 at\% $\mathrm{Al}, 10.2$ at\% $\mathrm{Zn}, 1.68$ at $\% \mathrm{Mg}$ alloy, as well as synchrotron radiation data obtained from individual grains extracted from a specially prepared alloy casting.

Structure analysis of the $\eta^{\prime}$-phase is met with several complications: Faults are frequent, composition and density are not known; the diffraction patterns are superpositions of four equivalent orientations of the hexagonal $\eta^{\prime}$-lattice relative to the aluminum matrix $\left(\mathrm{a}^{*}{ }_{\eta^{\prime}}=(1 / 3)[220]_{\mathrm{Al}} ; \mathrm{c}^{*}{ }_{\eta^{\prime}}=(1 / 6)[1-11]_{\mathrm{Al}}\right)$. The data are thus incomplete, due to overlap of reflections - and are in the electron case severely influenced by matrix-precipitate double diffraction. The latter effect was suppressed in our study, by the precession technique [3]. A three-dimensional data set to was collected by merging intensities from five projections. Synchrotron data from the same projections were extracted from a continuous three-dimensional intensity recorded on a MAR image plate.

Patterson and Fourier maps calculated from the incomplete intensity data obtained by either technique led to a structure model, in the space group, $\mathrm{P}_{3} \mathrm{~mm} / \mathrm{c}$ (194). Comparisons between selected area and precession electron diffraction with the synchrotron data will be presented.

[1] Auld J.H., Cousland S.McK., Scripta Met., 1971, 5, 765. [2] Li X.Z., Hansen V., Gjønnes J., Wallenberg L.R., Acta Mater., 1999, 47, 2651. [3] Vincent R., Midgley P.A., Ultramicroscopy, 1994, 53, 271.

Keywords: structure determination, electron diffraction, synchrotron radiation

\section{P.19.03.3}

Acta Cryst. (2005). A61, C458

Measurements of Valence Electron Distribution in Perovskite $\mathrm{CaCu}_{3} \mathrm{Ti}_{4} \mathrm{O}_{12}$

Yimei Zhu, J.C. Zheng, L. Wu, J. Hanson, W. Ku, Brookhaven National Laboratory, Upton, NY, 11973 USA. E-mail: zhu@bnl.gov

$\mathrm{CaCu}_{3} \mathrm{Ti}_{4} \mathrm{O}_{12}$ oxide recently has attracted much attention due to its extremely high dielectric constant $\left(\sim 10^{4}\right)$ from static fields up to kilohertz frequencies over a wide temperature range $(100 \sim 600 \mathrm{~K})$ [1]. In this presentation we report our study of the electronic structure and charge distribution of the material by combined use of quantitative electron diffraction, x-ray diffraction and DFT calculations. The electron diffraction was based on our unique diffraction-imaging coupled technique [2]: PArallel Recording Of Dark-field Images (PARODI). It is a new breed of convergent beam electron diffraction developed to accurately determine structure factors of low-order reflections that are sensitive to valence electron distribution. The synchrotron based single-crystal x-ray diffraction was mainly used to determine the structure factors of high-order reflections that are sensitive to atomic positions and Debye-Waller factors. The experimental results were then compared with various DFT calculations. Our results show that quantitative electron diffraction now can be used to test DFT. Combing the three techniques we are able to reveal valence electron diffraction, electron orbitals and bonding characteristics of complex functional materials.

This work is supported by U.S. DOE under Contract No. DE-AC02$76 \mathrm{CH} 00016$.

[1] Subramanian, et al., J. Sol. State Chem.,2000, 151, 323. [2] Wu L., et al, Phys. Rev. B, 2004, 69, 064501.

Keywords: charge density, convergent-beam electron diffraction, perovskite

\section{P.19.03.4}

Acta Cryst. (2005). A61, C458-C459

A Complex PbMnO $_{2.75}$ Phase solved by Electron Crystallography Holger Klein, Laboratoire de Cristallographie, 25 av. des Martyrs, 38042 Grenoble Cedex 9, France. E-mail: holger.klein@grenoble.cnrs.fr

When a crystalline structure has one small cell parameter, a high resolution electron micrograph (HREM) obtained in a transmission electron microscope (TEM) can give valuable information on the crystalline structure. Such an image can be seen as the convolution of the projection of the crystal potential and the microscope inherent contrast transfer function (CTF). After deconvolution a projected potential map is obtained. Especially in cases where the other cell parameters are very large and X-ray powder diffraction is not able to solve the structure this method is valuable.

In this contribution we will present the structure determined by the above method of a complex phase of approximate stoichiometry of $\mathrm{PbMnO}_{275}$. Its unit cell is monoclinic of space group $A 2 / m(a=$ $32.232 \AA, b=3.831 \AA, c=35.671 \AA, \beta=130^{\circ}$ ) [1].

Image treatment was done using the Visual Electron Crystallography software [2]. The images were first Fourier filtered, then deconvoluted from the contrast transfer. The obtained projected potential map allowed to distinguish the $\mathrm{Pb}$ and $\mathrm{Mn}$ cations and to place them in the projection onto the ac plane. Due to the small $b$ parameter and the space group, atoms can only be located at $y=0$ or $y$ $=0.5$. Taking into account the usual distances between cations in the same system allows to distinguish between the two values.

The final atomic coordinates differ little from the positions given 


\section{ELECTRON DIFFRACTION}

in [1]. The mean distance between the atoms is $0.3 \AA$, which is comparable to the standard deviation of the positions in [1].

[1] Bougerol C., Gorius M.-F., Grey I.E., JSSC, to be published. [2] http://cryst.iphy.ac.cn/VEC/

Keywords: electron crystallography, perovskite, HREM

P.19.04.1

Acta Cryst. (2005). A61, C459

Effect of Sonication and Grinding on the Structure of Amorphous

Carbon

Prabal Dasgupta ${ }^{\mathrm{a}}$, S. Chakraborty ${ }^{\mathrm{b}}$, Chitra Samanta ${ }^{\mathrm{c}},{ }^{a}$ C.S.S. Dept. ${ }^{b}$ MLS Unit, Indian Association for the Cultivation of Science, Kolkata.

'Jadavpur university, Kolkata, India. E-mail:

prabaldasgupta@hotmail.com

This work reports that on prolong sonication (3hr) and on grinding $(30 \mathrm{~min})$ the structure of freshly prepared hydrogenated amorphous carbon obtained by the pyrolysis of benzene changes considerably.

The $\mathrm{d}_{002}$ line particularly changes from $3.28 \mathrm{~A}^{\circ}$ to $3.81 \mathrm{~A}^{\circ}$ (sonication) and to $3.65 \mathrm{~A}^{\circ}$ (grinding), evident from electron diffraction studies. $\mathrm{d}_{002}$ line is also broadened in both the cases. FTIR studies reveal that on both the cases $\mathrm{sp}^{3} \mathrm{CH}_{2}$ (sym) and $\mathrm{sp}^{3} \mathrm{CH}_{2}$ (asym) streching modes at 2920 and $2850 \mathrm{~cm}^{-1}$ respectively shrinks, suggesting depletion of aliphatic hydrogen. On the other hand 1600 $\mathrm{cm}^{-1}$ band assigned to aromatic ring streching becomes more prominent in both the cases, suggesting increase in aromatic carbon content.

Keywords: electron diffraction, hydrogenated amorphous carbon, sonication 